\title{
Comparison of Hemoglobin Alc, Glycated Albumin and Fasting Plasma Glucose for Prediction of Arterial Stiffness in Chinese Adults
}

This article was published in the following Dove Press journal:

Diabetes, Metabolic Syndrome and Obesity: Targets and Therapy

\author{
Jianghua Wen ${ }^{1, *}$ \\ Fang $\mathrm{Hu}\left(\mathbb{D}^{2}{ }^{2} *\right.$ \\ Qiong Yang ${ }^{3}$
}

'Department of Health Examination, Jiangmen Central Hospital, Jiangmen 529070, Guangdong, People's Republic of China; ${ }^{2}$ Department of Endocrinology, The Fifth Affiliated Hospital, Sun Yat-sen University, Zhuhai 519000, Guangdong, People's Republic of China; ${ }^{3}$ Department of Endocrinology, Guilin Medical University Affiliated Hospital, Guilin 54I00I, Guangxi, People's Republic of China

*These authors contributed equally to this work
Correspondence: Qiong Yang

Department of Endocrinology, Guilin Medical University Affiliated Hospital, Guilin 54I00I, Guangxi, People's Republic of China

Tel +8613635189559

Fax +8607732823984

Email63269961@qq.com
Objective: Although diabetes is closely related to cardiovascular disease, there are some disputes whether diabetes can promote arterial stiffness. Therefore, the objective of this study is to compare the predictive abilities of related-glycemic markers including fast plasma glucose (FPG), glycated hemoglobin (HbAlc) and glycated albumin (GA) for the arterial stiffness.

Methods: In the present study, 3640 subjects (2171 men, 1469 women) were enrolled, and anthropometrics, brachial ankle pulse wave velocity (baPWV) and other laboratory data were obtained. Spearman correlation and multivariate logistic regression analyses were used to evaluate the relationships between FPG, HbAlc, GA and baPWV.

Results: Age, BMI, blood pressure, blood lipids, $\gamma$-Glutamyl transpeptidase, uric acid, hypersensitive C-reactive protein, baPWV, FPG, HbAlc, GA, estimated glomerular filtration rate and the incidences of diabetes and hypertension in high baPWV group were much greater than those in control group. Moreover, these above three glycemic markers were positively related to baPWV, and the correlation coefficient of HbAlc was the highest. After adjusting the above factors, HbAlc and FPG, but not GA, were still positively associated with baPWV regardless of diabetes status.

Conclusion: Our data demonstrated that, regardless of diabetes status, HbAlc and FPG were superior to GA for predicting arterial stiffness and HbAlc had the highest correlation with arterial stiffness, revealing that HbAlc may be regarded as an early diagnosis marker for atherosclerosis.

Keywords: diabetes, glycemic markers, cardiovascular disease, brachial ankle pulse wave velocity

\section{Introduction}

Cardiovascular diseases (CVD) are a leading reason to cause death worldwide, so it is very essential to identify their risk factors at the early stage of life. Arterial stiffness, the speed at which the pulse wave travels along a length of artery, as measured by noninvasive methods including brachial-ankle pulse wave velocity (baPWV), is widely considered as a well-established marker for atherosclerosis, and is also a strong marker for predicting the mortality and all-cause mortality of cardiovascular diseases. ${ }^{1}$

A global study concluded that higher than optimum blood glucose is a leading cause of cardiovascular mortality in most world regions, ${ }^{2}$ but the effect of glycemic control on arterial stiffness in the general population is unclear. Previous studies 
reported the positive relationships between glycemic markers including fast plasma glucose (FPG), glycated hemoglobin (HbAlc) and glycated albumin (GA), and arterial stiffness in the patients suffered from diabetes and chronic kidney diseases. ${ }^{3,4}$ To date, most studies reported the relationships between single glycemic marker and arterial stiffness, but few studies compared the predictive abilities of these three glycemic markers for arterial stiffness. ${ }^{5,6}$

Here, the associations of FPG, HbAlc and GA levels with baPWV in the Chinese population stratified by diabetes status were assessed, to identify which glycemic marker is more useful for early screening of atherosclerosis.

\section{Materials and Methods}

\section{Participants}

A total of 3976 subjects visiting the Health Examination Centre of Jiangmen Central Hospital (Guangdong, China) for a health checkup from May 2014 to April 2018 were enrolled, and the related clinical data, such as age, sex, body weight, height, disease history were obtained. Diabetes was defined as fasting glucose concentration $\geq 7.0 \mathrm{mmol} / 1$ or current use of hypoglycemic agents. In order to enlarge the sample size, those patients who received drug treatments for diabetes and hypertension were still enrolled in this study. However, the subjects suffered from infection, renal diseases (creatinine $\geq 150$ $\mu \mathrm{mol} / \mathrm{L}$ ), hepatic dysfunction [alanine aminotransferase (ALT) $\geq 3 \mathrm{x}$ ULN or aminotransferase (AST) $\geq 3 \mathrm{x}$ ULN], myocarditis and malignant diseases (diagnosed by clinical history or examinations) were excluded in our study. Finally, 3640 subjects consisting of 2171 men and 1469 women were included. The study was approved by the ethics committee of Jiangmen Central Hospital. Written informed consent was obtained for each participant, and that this study was conducted in accordance with the Declaration of Helsinki.

\section{Data Collection}

After fasting for at least $10 \mathrm{hrs}$ overnight, all the subjects were evaluated. In brief, BMI was calculated as the ratio of body weight to the square of height $\left(\mathrm{kg} / \mathrm{m}^{2}\right)$. HbAlc level was detected by immunoassay using high-performance liquid chromatography (Bio-Rad Laboratories, Munich, Germany). GA level was detected using a Hitachi 7600 autoanalyzer (Hitachi, Tokyo, Japan), and the result was presented as its percentage in total albumin. Serum total cholesterol (TC), high-density lipoprotein cholesterol
(HDL-C), low-density lipoprotein cholesterol (LDL-C), triglyceride (TG), FPG, AST, ALT, $\gamma$-Glutamyl transpeptidase $(\gamma$-GTP), creatinine, uric acid and hypersensitive $\mathrm{C}$-reactive protein (hsCRP) were also detected by a Hitachi 7600 autoanalyzer. The modified renal disease formula was used to determine the estimated glomerular filtration rate (eGFR).

\section{Determination of baPWV}

A non-invasive vascular screening device (VP-1000; Colin, Komaki, Japan) was applied for determining baPWV, estimating the arterial stiffness. All the subjects were in supine position; the electrocardiographic electrodes were placed on both wrists; pneumatic cuffs were placed on both arms and ankles; and a microphone was placed on the left edge of the sternum to determine heart sounds. Moreover, all patients' blood pressure and pulse volumes from bilateral arm and ankle were detected and recorded. As mentioned above, baPWV was bilaterally detected, but only a higher baPWV was used for statistical analysis. The cut-off value of high baPWV was more than $1400 \mathrm{~cm} / \mathrm{sec}$ in this study.

\section{Statistical Analysis}

All the statistical analyses were performed by SPSS (v. 16.0, Chicago, USA), and $P<0.05$ was regarded as statistical significance. Data at normal distribution were presented as mean $\pm \mathrm{SD}$, while data at skewed distribution were shown as median and interquartile range, and they were analyzed by $t$ test and MannWhitney $U$-test, respectively. Spearman correlation analysis was used to evaluate the correlations between FPG, HbAlc, GA and other indexes. Then, the participants were divided into groups with or without diabetes. Multivariate logistic regression analysis was carried out to evaluate the relationship between variables and the prevalence of arterial stiffness, and the result was presented as odds ratio (OR) with $95 \%$ confidence interval $(\mathrm{CI})$.

\section{Results}

\section{Clinical Characteristics of the Control and High baPWV Groups}

As presented in Table 1, the clinical characteristics of the control and high baPWV groups were compared, and the results indicated that the levels of age, BMI, blood pressure, TC, LDL-C, TG, ALT, AST, $\gamma$-GTP, hsCRP and uric acid, as 
Table I Comparisons of Clinical Parameters Between Control and High baPWV Groups

\begin{tabular}{|c|c|c|c|}
\hline Variables & $\begin{array}{l}\text { Control } \\
(n=2583)\end{array}$ & $\begin{array}{l}\text { High baPWV } \\
(n=1057)\end{array}$ & $\mathbf{P}$ \\
\hline Age (years) & $41.94 \pm 9.34$ & $53.34 \pm 10.59$ & $<0.001$ \\
\hline Body mass index $(\mathrm{kg} / \mathrm{m} 2)$ & $23.79 \pm 3.55$ & $24.85 \pm 3.27$ & $<0.001$ \\
\hline Male & $1492(57.76 \%)$ & 679 (64.24\%) & $<0.001$ \\
\hline Systolic blood pressure $(\mathrm{mm} \mathrm{Hg})$ & $115.36 \pm 11.20$ & $137.90 \pm 16.83$ & $<0.001$ \\
\hline Diastolic blood pressure $(\mathrm{mm} \mathrm{Hg})$ & $69.74 \pm 9.53$ & $84.13 \pm 11.51$ & $<0.001$ \\
\hline Fasting plasma glucose $(\mathrm{mmol} / \mathrm{L})$ & $5.23 \pm 1.08$ & $6.01 \pm 2.32$ & $<0.001$ \\
\hline Glycated hemoglobin (\%) & $5.67 \pm 0.67$ & $6.19 \pm 1.31$ & $<0.001$ \\
\hline Glycated albumin (\%) & $13.80 \pm 2.55$ & $15.02 \pm 4.80$ & $<0.001$ \\
\hline $\begin{array}{l}\text { Hypersensitive } \\
\text { C-reactive protein }(\mathrm{mmol} / \mathrm{l})\end{array}$ & $3.0(1.2-5.1)$ & $3.4(1.1-5.98)$ & $=0.004$ \\
\hline Total cholesterol (mmol/L) & $5.33 \pm 1.05$ & $5.69 \pm 1.17$ & $<0.001$ \\
\hline $\begin{array}{l}\text { Low-density lipoprotein } \\
\text { cholesterol (mmol/L) }\end{array}$ & $3.29 \pm 0.91$ & $3.50 \pm 1.03$ & $<0.001$ \\
\hline Triglycerides (mmol/L) & $1.28(0.87-1.94)$ & $1.72(1.17-2.59)$ & $<0.001$ \\
\hline $\begin{array}{l}\text { High-density lipoprotein } \\
\text { cholesterol (mmol/L) }\end{array}$ & $1.34 \pm 0.31$ & $1.33 \pm 0.30$ & $=0.187$ \\
\hline Alanine aminotransferase (U/L) & $19(13-30)$ & $23(16-34)$ & $<0.001$ \\
\hline Aspartate aminotransferase $(\mathrm{U} / \mathrm{L})$ & $21(17-26)$ & $27(23-32)$ & $<0.001$ \\
\hline Gamma-glutamyltransferase (U/L) & $23(16-37)$ & $29(20-44)$ & $<0.001$ \\
\hline Uric acid (mg/dl) & $\begin{array}{l}357.1 \\
(290.0-430.0)\end{array}$ & $\begin{array}{l}379.1 \\
(312.4-450.0)\end{array}$ & $<0.001$ \\
\hline $\begin{array}{l}\text { Estimated glomerular filtration } \\
\text { rate }\end{array}$ & $\begin{array}{l}93.00 \\
(81.04-107.72)\end{array}$ & $\begin{array}{l}83.09 \\
(69.67-98.63)\end{array}$ & $<0.001$ \\
\hline Diabetes & 115 (4.45\%) & $204(19.30 \%)$ & $<0.001$ \\
\hline Hypertension & $57(2.21 \%)$ & $211(19.96 \%)$ & $<0.001$ \\
\hline
\end{tabular}

Abbreviation: baPWV, brachial-ankle pulse wave velocity.

well as the incidences of diabetes and hypertension, were higher, but eGFR was lower in the high baPWV group relative to the control group $(P<0.05)$. No significant difference in HDL-C was observed between these above two groups. Moreover, the high baPWV group demonstrated obvious increases in three glycemic markers including FPG, HbAlc and GA, compared to the control group $(P<0.05)$.

\section{Correlation Between FPG, HbAlc, GA and Other Risk Factors}

Spearman's rank correlation analysis demonstrated that HbAlc and FPG were positively and significantly associated with age, anthropometric measures such as baPWV, BMI, systolic BP, diastolic BP and biochemical indexes such as TC, LDL-C, TG, ALT, $\gamma$-GTP, hsCRP, uric acid. GA was also positively and significantly associated with baPWV and age, but negatively and significantly associated with BMI, TC, LDL-C, TG, ALT, AST, $\gamma$-GTP, hsCRP, uric acid and eGFR. In addition, HbAlc and FPG were negatively and significantly related to HDL-C, while GA was positively associated with HDL-C (Table 2).

\section{Multiple-Factor Analysis of Relationships Among $\mathrm{HbAlc}$, GA, FPG and Arterial Stiffness}

When the participants were divided based on diabetes status, multivariate logistic regression analyses of the relationships among HbA1c, GA, FPG and arterial stiffness were reassessed in each group, and the results are presented in Table 3. After adjustment for conventional risk factors, such as age, BMI, sex, BP, TC, TG, HDL-C, LDLC, ALT, AST, $\gamma$-GTP, hsCRP, uric acid, eGFR, the incidences of hypertension, arterial stiffness was significantly and independently predicted by HbAlc and FPG in both diabetic and non-diabetic subjects $(P<0.05$ for all). However, there was no significant association between GA and arterial stiffness in both diabetic and nondiabetic subjects.

\section{Discussion}

In this study, we compared the predictive abilities of HbAlc, GA and FPG for the arterial stiffness measured by baPWV in general adults. The main findings were that, even after controlling for other confounding factors, HbAlc was closely related to arterial stiffness with the highest correlation in subjects with or without diabetes, followed by FPG. Interestingly, there was no independent correlation between GA and baPWV. These results imply that HbAlc and FPG are superior to GA for predicting arterial stiffness, and HbAlc may be an early diagnosis marker for atherosclerosis.

Arterial stiffness was proved to have a key and independent predictive ability for cardiovascular mortality, coronary events and atherosclerotic diseases. ${ }^{7,8}$ Therefore, it is essential to earlier determine the arterial stiffness in primary and secondary preventions of major CVD. Several studies have shown that baPWV was used broadly in China and Japan, and was generally accepted as the most simple, noninvasive and reproducible parameter for arterial stiffness, and was closely related to aortic PWV measured by invasive catheter manometer. ${ }^{2,9}$ So, patients with anomalous baPWV were most likely to present early-stage atherosclerosis. In our study, high baPWV was defined as greater than $1400 \mathrm{~cm} /$ sec since the cut-off point was an independent variable for the risk stratification of patients with atherosclerotic cardiovascular diseases based on Framingham score. ${ }^{10}$

Our results were consistent with previous studies demonstrating that elevated HbAlc level was markedly associated with enhanced arterial stiffness determined by baPWV, and was independent from other cardiovascular risk factors. Some 
Table 2 Spearman's Rank Correlations Between Three Glycemic Markers and Clinical Parameters

\begin{tabular}{|c|c|c|c|c|c|c|}
\hline Variables & FPG & $\mathbf{P}$ & HbAlc & $\mathbf{P}$ & GA & $\mathbf{P}$ \\
\hline Age & 0.278 & $<0.001$ & 0.406 & $<0.001$ & 0.305 & $<0.001$ \\
\hline Body mass index & 0.256 & $<0.001$ & 0.307 & $<0.001$ & -0.271 & $<0.001$ \\
\hline Systolic blood pressure & 0.314 & $<0.001$ & 0.323 & $<0.001$ & 0.026 & $=0.111$ \\
\hline Diastolic blood pressure & 0.298 & $<0.001$ & 0.318 & $<0.001$ & 0.004 & $=0.805$ \\
\hline Hypersensitive C-reactive protein & 0.084 & $<0.001$ & 0.176 & $<0.001$ & -0.039 & $=0.019$ \\
\hline Total cholesterol & 0.168 & $<0.001$ & 0.241 & $<0.001$ & -0.047 & $=0.004$ \\
\hline Low-density lipoprotein cholesterol & 0.126 & $<0.001$ & 0.253 & $<0.001$ & -0.072 & $<0.001$ \\
\hline Triglycerides & 0.221 & $<0.001$ & 0.273 & $<0.001$ & -0.193 & $<0.001$ \\
\hline High-density lipoprotein cholesterol & -0.106 & $<0.001$ & -0.116 & $<0.001$ & 0.162 & $<0.001$ \\
\hline Alanine aminotransferase & 0.153 & $<0.001$ & 0.148 & $<0.001$ & -0.212 & $<0.001$ \\
\hline Aspartate aminotransferase & 0.046 & $=0.005$ & 0.021 & $=0.205$ & -0.071 & $<0.001$ \\
\hline Gamma-glutamyltransferase & 0.212 & $<0.001$ & 0.220 & $<0.001$ & -0.221 & $<0.001$ \\
\hline Uric acid & 0.106 & $<0.001$ & 0.125 & $<0.001$ & -0.237 & $<0.001$ \\
\hline Estimated glomerular filtration rate & 0.011 & $=0.534$ & 0.036 & $=0.035$ & -0.334 & $<0.001$ \\
\hline Brachial-ankle pulse wave velocity & 0.313 & $<0.001$ & 0.355 & $<0.001$ & 0.115 & $<0.001$ \\
\hline
\end{tabular}

Abbreviations: FPG, fasting plasma glucose; HbAlc, glycated hemoglobin; GA, glycated albumin.

Table 3 Multivariate Logistic Regression Analyses for Three Glycemic Markers with High baPWV in Non-Diabetic Subjects and Diabetic Subjects

\begin{tabular}{|l|l|l|l|l|}
\hline Variables & \multicolumn{2}{|l|}{$\begin{array}{l}\text { Non-Diabetic Subjects } \\
(\mathbf{n}=332 \mathrm{I})\end{array}$} & \multicolumn{2}{l|}{$\begin{array}{l}\text { Diabetic Subjects } \\
\text { (n=319) }\end{array}$} \\
\cline { 2 - 5 } & OR (95\% Cl) & P & OR (95\% Cl) & P \\
\hline FPG & $1.45(1.14-1.84)$ & 0.003 & $1.15(1.03-1.28)$ & 0.014 \\
HbAlc & $1.54(1.09-2.20)$ & 0.016 & $1.31(1.06-1.62)$ & 0.014 \\
GA & $0.96(0.87-1.06)$ & 0.414 & $1.03(0.98-1.08)$ & 0.225 \\
\hline
\end{tabular}

Abbreviations: FPG, fasting plasma glucose; HbAlc, glycated hemoglobin; GA, glycated albumin; baPWV, brachial-ankle pulse wave velocity.

studies on Asian population demonstrated that high level of HbAlc was significantly and independently associated with enhanced baPWV in Chinese and Japanese, ${ }^{11,12}$ and similar findings were also found in Western population. ${ }^{13}$ Our study further confirmed that HbAlc was superior to FPG and GA for predicting arterial stiffness in general Chinese population, regardless of whether or not in those with a normal glucose status. HbAlc, regarded as a precursor of increased advanced glycation end products (AGEs), may be related to the amount of AGEs. ${ }^{14}$ Evidences indicated that the increased AGEs were associated with the diabetes-related complications and vascular diseases. ${ }^{15}$ The mechanisms affecting arterial stiffness included activating pro-inflammatory cell signaling and upregulating NADPH oxidase and adhesion molecules, as well as promoting the proliferation of vascular smooth muscle cells. ${ }^{3,16,17}$ Furthermore, AGEs could form cross-links in collagen fibers and decrease the distensibility of arterial walls, directly damaging the artery. $^{18}$ The above-mentioned mechanisms revealed that AGEs might have important effects on the early progression of atherosclerosis. Moreover, Saha and Schwarz reported that $\mathrm{HbA1c}$ may be a clinically useful and simple index for predicting the concomitant presence of insulin resistance among apparently healthy individuals. ${ }^{19}$ Insulin resistance also has been proven to increase arterial stiffness. ${ }^{20}$ So, high level of HbAlc may be helpful for increasing baPWV via insulin resistance.

GA, an early Amadori-type glycation protein of the nonenzymatic glycation reaction between glucose and albumin, reflects 2-3 weeks shorter-term glycemic control compared with HbAlc. $^{21}$ Therefore, GA was considered more useful than HbAlc for evaluating shorter-term changes of glucose. However, there were some disputes about the relationship between GA level and CVD. Kumeda et al proposed that GA was an independent factor to predict arterial stiffness in patients suffered with type 2 diabetes. $^{22}$ In a study by Zeng et $\mathrm{al},{ }^{6}$ both GA and HbA1c were associated with arterial stiffness, but the study did not adjust for $\gamma$-GTP and uric acid, which had been reported as independent predictors of arterial stiffness. $^{23,24}$ On the contrary, the recently published Diabetes Control and Complications Trial (DCCT)/ Epidemiology of Diabetes Interventions and Complications study failed to demonstrate a significant association of GA and CVD events in subjects with type 1 diabetes mellitus (T1DM). ${ }^{25}$ In the present study, GA was not proved to be an independent predictive index for arterial stiffness after adjusting potential confounders in subjects with or without diabetes. These reports imply that there is only a weak relationship between GA level and atherosclerosis. One of the 
possible reasons to cause this phenomenon is that serum GA was negatively correlated with BMI, hsCRP, atherogenic dyslipidemia indexes including TC, LDL-C and TG, but was positively and significantly associated with HDL-C. Furthermore, chronic micro-inflammation could also lead to accelerated albumin catabolism, and thereby causing the decrease in GA level. ${ }^{26}$ All these reasons together accounted for the weak correlation of GA level with arterial stiffness in this study.

Earlier studies have indicated that high level of FPG was a risk indicator for CVD in type 2 diabetes patients. $^{27,28}$ Whereas, few related literatures that explored the roles that FPG played in early-stage atherosclerosis in the subjects without diabetes were reported. According to our study, FPG was independently and positively related to baPWV after adjusting confounding factors in the subjects with or without diabetes. Thus, this finding implies that high level of glucose within normal range can be also helpful for increasing the incidence of arterial stiffness.

Of course, there were several limitations in this study. Firstly, our study only included cross-sectional data and correlation analysis, but no causal correlation of glycemic markers with the risk factors for arterial stiffness was demonstrated. Secondly, although there was a large-size sample, we did not obtain complete data on CVD risk factors, such as alcohol consumption and smoking status. In the end, the relationships between glycemic markers and arterial stiffness measured by other atherosclerosis markers, such as carotid-femoral PWV or carotid intimamedia thickness, were not assessed.

Collectively, our data demonstrated that HbAlc and FPG had a better predictive ability for arterial stiffness than GA, and HbAlc had the highest association with arterial stiffness, regardless of diabetes status in general Chinese adults, revealing that HbAlc may be used as an early diagnosis marker for atherosclerosis. However, more prospective studies are still needed to further verify this conclusion.

\section{Acknowledgments}

The project was supported by Self-funded project of district health and fitness committee of Guangxi Province (Z20190158).

\section{Disclosure}

All authors declare that there are no conflicts of interest.

\section{References}

1. Yamashina A, Tomiyama H, Takeda K, et al. Validity, reproducibility, and clinical significance of noninvasive brachial-ankle pulse wave velocity measurement. Hypertens Res. 2002;25:359-364. doi:10.12 91/hypres.25.359

2. Danaei G, Lawes CM, Hoorn SV, et al. Global and regional mortality from ischaemic heart disease and stroke attributable to higher-thanoptimum blood glucose concentration: comparative risk assessment. Lancet (North American Edition). 2006;368(9548):0-1659.

3. Teoh WL, Price JF, Williamson RM, et al. Metabolic parameters associated with arterial stiffness in older adults with type 2 diabetes: the Edinburgh type 2 diabetes study. $J$ Hypertens. 2013;31:1010-1017. doi:10.1097/HJH.0b013e32835f7ecf

4. Choi HY, Park SK, Yun GY, et al. Glycated albumin is independently associated with arterial stiffness in non-diabetic chronic kidney disease patients. Medicine (Baltimore). 2016;95:e3362. doi:10.1097/ MD.0000000000003362

5. Mukai N, Ninomiya T, Hata J, et al. Association of hemoglobin Alc and glycated albumin with carotid atherosclerosis in community-dwelling Japanese subjects: the Hisayama Study. Cardiovasc Diabetol. 2015;14:84. doi:10.1186/s12933-015-0247-7

6. Zeng Q, Dong SY, Wang ML, et al. Serum glycated albumin, glycated hemoglobin, and arterial stiffness in a general Chinese population. Clin Chim Acta. 2017;468:33-38. doi:10.1016/j.cca.2017.02.002

7. Ninomiya T, Kojima I, Doi Y, et al. Brachial-ankle pulse wave velocity predicts the development of cardiovascular disease in a general Japanese population: the Hisayama Study. J Hypertens. 2013;31:477-483; discussion 483 .

8. Boutouyrie P, Tropeano AI, Asmar R, et al. Aortic stiffness is an independent predictor of primary coronary events in hypertensive patients: a longitudinal study. Hypertension. 2002;39:10-15. doi:10.1 161/hy0102.099031

9. Lu Y C, Lyu P, Zhu H Y, et al. Brachial-ankle pulse wave velocity compared with mean arterial pressure and pulse pressure in risk stratification in a Chinese population. $J$ Hypertens. 2018;36:1.

10. Yamashina A, Tomiyama H, Arai T, et al. Brachial-ankle pulse wave velocity as a marker of atherosclerotic vascular damage and cardiovascular risk. Hypertens Res. 2003;26:615-622. doi:10.1291/ hypres. 26.615

11. Hung CS, Lee PC, Li HY, et al. Haemoglobin A1c is associated with carotid intima-media thickness in a Chinese population. Clin Endocrinol. 2011;75:780-785. doi:10.1111/j.1365-2265.2011.04129.x

12. Venkataraman V, Amutha A, Anbalagan VP, et al. Association of glycated hemoglobin with carotid intimal medial thickness in Asian Indians with normal glucose tolerance. $J$ Diabetes Complications. 2012;26:526-530. doi:10.1016/j.jdiacomp.2012.06.002

13. Haring R, SE B, Lieb W, et al. Glycated hemoglobin as a marker of subclinical atherosclerosis and cardiac remodeling among non-diabetic adults from the general population. Diabetes Res Clin Pract. 2014;105:416-423. doi:10.1016/j.diabres.2014.05.004

14. Makita Z, Vlassara H, Rayfield E, et al. Hemoglobin-AGE: a circulating marker of advanced glycosylation. Science. 1992;258:651-653.

15. Goldin A, Beckman JA, Schmidt AM, Creager MA. Advanced glycation end products: sparking the development of diabetic vascular injury. Circulation. 2006;114:597-605. doi:10.1161/CIRCULATION AHA.106.621854

16. Paradela-Dobarro B, Rodino-Janeiro BK, Alonso J, et al. Key structural and functional differences between early and advanced glycation products. J Mol Endocrinol. 2016;56:23-37. doi:10.1530/JME-150031

17. Nambiar S, Viswanathan S, Zachariah B, Hanumanthappa N, Agrawal A, Nadaradjan RD. Possible modulation of glycated protein levels in prehypertension by lipid peroxides. Clin Exp Hypertens. 2012;34:517-522. doi:10.3109/10641963.2012.681721 
18. Schram MT, Henry RM, van Dijk RA, et al. Increased central artery stiffness in impaired glucose metabolism and type 2 diabetes: the Hoorn study. Hypertension. 2004;43:176-181. doi:10.1161/01. HYP.0000111829.46090.92

19. Saha S, Schwarz PE. Impact of glycated hemoglobin (HbA1c) on identifying insulin resistance among apparently healthy individuals. $J$ Public Health (Bangkok). 2017;(suppl 1):1-8.

20. Meng C, Sun M, Wang Z, et al. Insulin sensitivity and beta-cell function are associated with arterial stiffness in individuals without hypertension. J Diabetes Res. 2013;2013:1-8.

21. Kim KJ, Lee BW. The roles of glycated albumin as intermediate glycation index and pathogenic protein. Diabetes Metab J. 2012;36:98-107. doi:10.4093/dmj.2012.36.2.98

22. Kumeda Y, Inaba M, Shoji S, et al. Significant correlation of glycated albumin, but not glycated haemoglobin, with arterial stiffening in haemodialysis patients with type 2 diabetes. Clin Endocrinol (Oxf). 2010;69(4):556-561. doi:10.1111/j.1365-2265.2008.03202.X

23. Jung $\mathrm{CH}, \mathrm{J}$ H Y, Bae SJ, et al. Serum gamma-glutamyltransferase is associated with arterial stiffness in healthy individuals. Clin Endocrinol (Oxf). 2011;75(3):328-334. doi:10.1111/j.1365-2265.2011.04060.x
24. Kuo CF, Yu KH, Luo SF, et al. Role of uric acid in the link between arterial stiffness and cardiac hypertrophy: a cross-sectional study. Rheumatology. 2010;49(6):1189-1196. doi:10.1093/rheumatology/keq095

25. Nathan DM, McGee P, Steffes MW, Lachin JM. Relationship of glycated albumin to blood glucose and HbA1c values and to retinopathy, nephropathy, and cardiovascular outcomes in the DCCT/EDIC study. Diabetes. 2014;63:282-290. doi:10.2337/db13-0782

26. Koga M, Otsuki M, Matsumoto S, et al. Negative association of obesity and its related chronic inflammation with serum glycated albumin but not glycated hemoglobin levels. Clin Chim Acta. 2007;378(1-2):48-52. doi:10.1016/j.cca.2006.10.013

27. Group T D S. Is the current definition for diabetes relevant to mortality risk from all causes and cardiovascular and noncardiovascular diseases? Diabetes Care. 2003;26(3):688-696. doi:10.2337/diacare.26.3.688.

28. Eun YM, Kang SG, Song SW. Fasting plasma glucose levels and coronary artery calcification in subjects with impaired fasting glucose. Ann Saudi Med. 2016;36(5):334-340. doi:10.5144/02564947.2016.334

Diabetes, Metabolic Syndrome and Obesity: Targets and Therapy

\section{Dovepress}

\section{Publish your work in this journal}

Diabetes, Metabolic Syndrome and Obesity: Targets and Therapy is an international, peer-reviewed open-access journal committed to the rapid publication of the latest laboratory and clinical findings in the fields of diabetes, metabolic syndrome and obesity research. Original research, review, case reports, hypothesis formation, expert opinion and commentaries are all considered for publication. The manuscript management system is completely online and includes a very quick and fair peer-review system, which is all easy to use. Visit http://www.dovepress.com/testimonials.php to read real quotes from published authors.

Submit your manuscript here: https://www.dovepress.com/diabetes-metabolic-syndrome-and-obesity-targets-and-therapy-journal 